\title{
Modeling Energy Transfer in Quantum Thermal Machines
}

\section{A new modeling and computational approach allows for more complete simulations of particle and heat flow through tiny quantum devices.}

\section{By Amikam Levy and Wenjie Dou}

A system interacting with its surroundings exchanges energy in various forms, such as through the flow of heat Ior particles. These transport processes contain valuable information about both the system and surroundings. At the quantum scale, describing such non-equilibrium processes is relevant to understanding, for example, how charges flow through a semiconductor quantum dot or through a molecular junction (Fig. 1). But how do quantum phenomena govern energy transport processes, and how can the resulting effects be harnessed to develop novel quantum technologies?

Answering these questions theoretically is a very difficult task, particularly for a system that is strongly coupled to its surroundings. Marlon Brenes from Trinity College Dublin and colleagues now report a new tool for simulating the non-equilibrium dynamics of such strongly coupled systems [1].

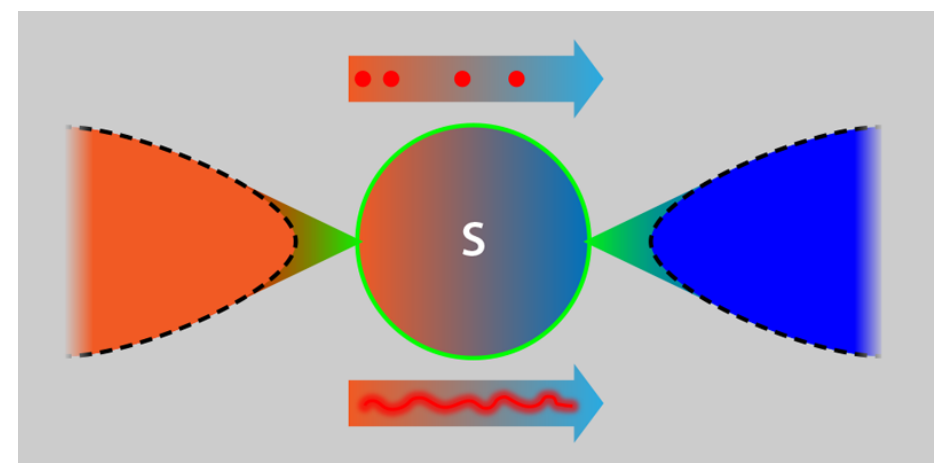

Figure 1: In a simple thermal machine, a system $\mathrm{S}$ is coupled to two reservoirs with different chemical potentials and temperatures. As a result of the chemical-potential difference, currents of particles and energy are sustained through the system. Credit: M. Brenes et al. [1]; adapted by APS/Alan Stonebraker
Significantly, the team's computational approach applies to a system of electrons whether the electrons' interactions with one another are weak or strong. Their method improves upon tools that work well for systems that interact only weakly with their surroundings, and it opens new possibilities for exploring thermal machines at the quantum scale, which are now being developed in the lab.

Quantum effects typically only show up in a thermal machine if it's at most a few hundred atoms in size. Until recently, such machines were purely theoretical. But thanks to ongoing advances in nanofabrication and the control of small quantum systems, experimentalists can now create tiny thermodynamic machines that are subject to the rules of quantum mechanics and that play an integral role in the development of new quantum technologies.

So far, most studies of quantum thermal machines have focused on systems that interact weakly with their surroundings [2], such as atoms and molecules that interact with electromagnetic radiation. The weak coupling assumption allows for a clear separation between the system and its environment, simplifying the description of the system. Moreover, the system-environment separation means that it is possible to define thermodynamic processes, such as heat flows, simply in terms of the system.

However, this clear separation is no longer justified in a system that's strongly coupled to its environment. Studies of such a system's behavior must instead explicitly incorporate information about the coupling and about the environment [3], which adds multiple degrees of freedom to the calculations and demands great computational effort. Researchers have 
developed numerous methods to address this problem. One of the most important contributions dates to around 1960, when Sadao Nakajima, Robert Zwanzig, and others derived a "generalized quantum master equation" to describe the dynamics of many-particle systems $[4,5]$. In this approach, information about the environment and its coupling to the system have to be separately maintained and updated throughout the calculation. To avoid this complication, many of the current methods strongly couple the system to a finite bath, which in turn is weakly coupled to a macroscopic environment. The equations that describe the dynamics of the system and its interactions with the intermediate or "primary" bath are then solved explicitly. This idea has been implemented into various theoretical methods that are used to describe quantum thermal machines [6-8].

Brenes and co-workers take a similar path, but with novel applications of the tools for modeling the environment [1]. In their picture, a simple thermal machine or electronic system is coupled to a finite number of mesoscopic metal leads that, collectively, represent the primary bath (Fig. 2). Each lead serves as an electronic mode with a well-defined frequency, and it is continuously damped toward thermal equilibrium by a weak coupling to a macroscopic environment. The researchers then write a master equation in terms of the parameters that describe the leads and apply it to two types of systems.

For systems in which the electrons do not interact with each other, the team shows they can recast their equation into a different form using the so-called superfermionic representation [9]. This transformation doubles the overall system size, which might seem like a disadvantage. But it actually simplifies calculations of steady-state transport properties because the calculations can be done with standard methods. The numerical solution to the master equation converges for a relatively small number of lead sites and is used to determine the heat and particle flow at steady state.

The team also considers interacting electronic systems, which pose even more interesting and challenging problems. Up until now, only a small number of studies have considered quantum thermodynamics in regimes that have both strong electron-electron interactions and strong system-bath coupling $[7,8]$. Brenes and co-workers tackle this difficult combination. To do so, they describe the system using a so-called tensor

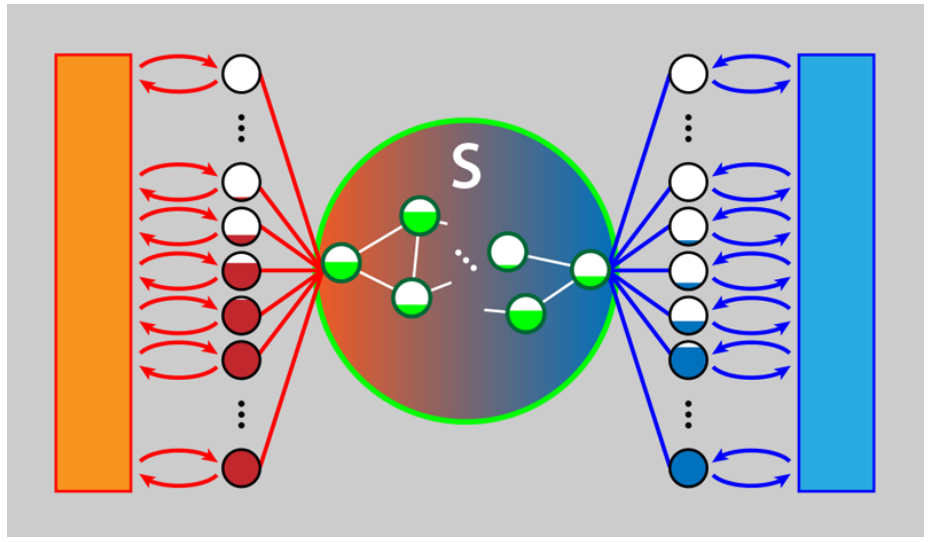

Figure 2: Brenes and co-workers approminate the system's connection to the environment in terms of a collection of mesoscopic leads. Using this model, the equations that describe the system's dynamics may be more easily solved.

Credit: M. Brenes et al. [1]; adapted by APS/Alan Stonebraker

network. This mathematical tool has become popular for studying strongly interacting systems, but it typically works well only when those interactions are short ranged. The researchers ensure that their use of tensor networks applies to both shortand long-ranged interacting systems. Specifically, they again use the super-fermionic representation and a set of mappings to reformulate their master equation, which eliminates the long-ranged interactions in the description of the leads. Using this scheme, the researchers study an autonomous quantum heat engine with three atoms that are strongly coupled to their environment. Interestingly, they find that the inclusion of electron-electron interactions can enhance the power and efficiency of the engine, a prediction that can potentially be tested in the lab.

Considering the primary bath and the system explicitly has become a prevalent way to account for strong system-environment coupling effects. The approach introduced by Brenes and colleagues is particularly valuable because it combines the novel use of mesoscopic leads with the ongoing development of efficient tensor network algorithms for open systems $[1,10,11]$. Looking forward, it would be of great interest to develop algorithms for system dynamics that incorporate time-dependent driving fields. In these cases, the interplay between work, heat, and particle flows becomes more involved. The driving Hamiltonian-or how fast the system's 
total energy changes in time-gives rise to new forms of irreversibility as well as the notion of quantum friction. These concepts are not only important from a theoretical perspective but are also very helpful in designing efficient transport processes in superconducting qubits, quantum dots, single-molecule junctions, and in many other quantum devices.

Amikam Levy: Department of Chemistry, University of California, Berkeley, CA, USA and Department of Chemistry, Bar-Ilan University, Ramat Gan, Israel

Wenjie Dou: Department of Chemistry, University of California, Berkeley, CA, USA and School of Science, Westlake University, Hangzhou, Zhejiang, China

\section{REFERENCES}

1. M. Brenes et al., "Tensor-network method to simulate strongly interacting quantum thermal machines," Phys. Rev. X 10, 031040 (2020).

2. R. Kosloff and A. Levy, "Quantum heat engines and refrigerators: Continuous devices," Annu. Rev. Phys. Chem. 65, 365 (2014).

3. M. Esposito et al., "Quantum thermodynamics: A nonequilibrium Green's function approach," Phys. Rev. Lett.
114, 080602 (2015).

4. S. Nakajima, "On quantum theory of transport phenomena: steady diffusion," Prog. Theor. Phys. 20, 948 (1958).

5. R. Zwanzig, "Ensemble method in the theory of irreversibility," J. Chem. Phys. 33, 1338 (1960).

6. G. Katz and R. Kosloff, "Quantum thermodynamics in strong coupling: Heat transport and refrigeration," Entropy 18, 186 (2016).

7. W. Dou et al., "Universal approach to quantum thermodynamics in the strong coupling regime," Phys. Rev. B 98, 134306 (2018).

8. W. Dou et al., "Universal approach to quantum thermodynamics of strongly coupled systems under nonequilibrium conditions and external driving," Phys. Rev. B 101, 184304 (2020).

9. A. A. Dzhioev and D. S. Kosov, "Super-fermion representation of quantum kinetic equations for the electron transport problem,” J. Chem. Phys. 134, 044121 (2011).

10. M. Lotem et al., "Renormalized Lindblad driving: A Numerically-exact nonequilibrium quantum impurity solver," arXiv:2004.07637.

11. G. Wójtowicz et al., "Open-system tensor networks and Kramers' crossover for quantum transport," Phys. Rev. A 101, $050301(2020)$. 